EPJ Web of Conferences 52, 01006 (2013)

DOI: $10.1051 /$ epjconf/20135201006

(C) Owned by the authors, published by EDP Sciences, 2013

\title{
Results from TOTEM
}

\author{
Karsten Eggert ${ }^{1, a}$ \\ ${ }^{1}$ Case Western Reserve University, Cleveland, Ohio, USA
}

\begin{abstract}
The TOTEM experiment at the CERN LHC is focussed on the measurement of the elastic proton-proton scattering, the total pp cross-section, and all kinds of diffractive phenomena. Detectors housed in "Roman Pots" which can be moved close to the outgoing proton beams allow to trigger on elastic and diffractive protons and to determine their parameters like the momentum loss and the transverse momentum transfer. In addition, charged particle detectors in the forward regions detect almost all inelastic events. Together with the CMS detector, a large solid angle is covered enabling precise studies of Min. Bias as well as Single Diffractive and Double Pomeron Interactions. The results will considerably help the interpretation of the Cosmic Ray Showers at highest energies and will give insight into the proton structure and the QCD theory of strong interactions. TOTEM measured the elastic pp- scattering over a large range of $t$ (the squared momentum transfer) from $10^{-3}-4 \mathrm{GeV}^{2}$. None of the considered models could yield a satisfactory fit over the complete range. However, the exponential slope at low $|\mathrm{t}|$-values and the position of the diffractive minimum are well within the extrapolation from lower energies. The total pp cross-section has been determined in different ways from the extrapolation of the elastic scattering to $t=0$ (optical point) and the inelastic rate: (i) From the elastic scattering using the optical theorem and the CMS, (ii) luminosity independently, using the inelastic rate, elastic scattering and the optical theorem, (iii) $\rho$ independently, by using elastic scattering, inelastic rate and the CMS luminosity. The results for the total crosssection obtained from the different methods are in excellent agreement with each other. First studies of the data on diffractive phenomena have been performed by correlating the momentum loss of the forward protons with the topology of the particle flux. The data look very promising and further studies will follow. Since the conference, data have also been taken with specialized optics to reach even lower $|\mathrm{t}|$-values. A determination of the $\rho$ value is now in reach. The TOTEM results and the ways how they have been obtained are described in the publications [1-8] listed below.
\end{abstract}

\section{References}

[1] The TOTEM Collaboration, 2008 JINST 3 S08007; doi:10.1088/1748-0221/3/08/S08007

[2] The TOTEM Collaboration, arXiv:1110.1385 ; TOTEM-2011-01 ; CERN-PH-EP-2011-101

[3] The TOTEM Collaboration, CERN-PH-EP-2011-158; EPL 96 (2011) 21002

[4] The TOTEM Collaboration, arXiv:1205.4105; CERN-PH-EP-2012-106; EPL 98 (2012) 31002

[5] The TOTEM Collaboration, CERN-PH-EP-2012-239

${ }^{\mathrm{a}}$ karsten.eggert@cern.ch 
[6] The TOTEM Collaboration, CERN-PH-EP-2012-352

[7] The TOTEM Collaboration, CERN-PH-EP-2012-353

[8] The TOTEM Collaboration, CERN-PH-EP-2012-354

\section{Slides}

The slides of the talk can be found on the website of the symposium ISVHECRI 2012: https://indico.desy.de/conferenceOtherViews.py?view=standard\&confId=4594 